\title{
La ciencia como un punto de vista: algunos desafíos a la objetividad científica*
}

\author{
Science as a Point of View: Some Challenges \\ to Scientific Objectivity
}

\author{
MARIO GENSOLLEN** \\ MARC JIMÉNEZ ROLLAND***
}

\begin{abstract}
Resumen: Algunos críticos de la ciencia afirman que es sólo un punto de vista entre otros, sin alguna autoridad epistémica especial. No obstante, en este artículo se defiende que la idea de que la investigación científica involucra una perspectiva o punto de vista no impone una restricción a su ideal de objetividad. Primero se presentan algunas aclaraciones sobre la noción de punto de vista, luego se atiende al concepto de objetividad científica, y por último se enfrentan algunos desafíos que se desprenden de la noción de punto de vista y amenazan rasgos de la objetividad científica vinculados con su autoridad epistémica.

Palabras clave: autoridad epistémica; perspectiva; compromisos normativos, subdeterminación.
\end{abstract}

\begin{abstract}
Some critics of science claim that it is only a point of view among others, lacking any special epistemic authority. However, this paper contends that conceiving scientific inquiry as something that involves a perspective or a point of view does not pose a constraint on its ideal of objectivity. We first put forward some clarifications on the notion of point of view, followed by some observations on scientific objectivity; finally, we face some of the challenges that rise from the notion of point of view and threaten features of scientific objectivity tied to its epistemic authority.
\end{abstract}

Keywords: epistemic authority; perspective; normative commitments, underdetermination.

Recibido: 30/06/2018. Aceptado: 21/10/2018.

* Este trabajo se enmarca en el proyecto de investigación "Epistemología aplicada" (PIF 18-2) financiado por la Universidad Autónoma de Aguascalientes.

** Profesor investigador Titular C en la Universidad Autónoma de Aguascalientes. E-mail: mgenso@gmail.com. Recientemente ha publicado "El lugar de la Teoría de la virtud argumentativa en la teoría de la argumentación contemporánea" (Revista Iberoamericana de Argumentación 15, 2017: 41-59), y coedita, junto con Anna Estany, el libro Democracia y conocimiento (Aguascalientes: Universitat Autònoma de Barcelona/Universidad Autónoma de Aguascalientes/IMAC, 2018). Su línea de investigación es la correlación entre Epistemología, Teoría de la argumentación y Filosofía política.

*** Profesor Titular B de Epistemología en la Universidad Autónoma de Aguascalientes. E-mail: marcji2121@ yahoo.com. Su trabajo "Conocimiento y justificación en la epistemología democrática" (en Democracia y conocimiento, Aguascalientes: Universitat Autònoma de Barcelona/Universidad Autónoma de Aguascalientes/ IMAC, 2018) se encuentra en prensa. Su línea de investigación es la aplicación de métodos formales a problemas filosóficos, en especial en las áreas de Filosofía de la ciencia, Epistemología y Filosofía del lenguaje. 


\section{Introducción}

La ciencia goza de amplia aceptación en nuestra sociedad por razones tanto teóricas como prácticas. En el plano teórico, “queremos aceptar sólo aquello para lo que hay buena razón para creer que es verdadero; y consideramos que la ciencia es la única buena manera de llegar a creencias razonables acerca de lo que es verdadero, al menos en el ámbito de lo puramente fáctico" (Boghossian, 2006, 4) ${ }^{1}$. Por esta razón, muchas personas intentan avalar sus prácticas y productos llamándolos 'científicos', con lo que buscan que las demás consideren su trabajo similar a la especial y fiable fuente de conocimiento que la ciencia dice brindarnos. Para algunas personas, la ciencia tiene una autoridad epistémica especial. Si la ciencia tiene esta autoridad epistémica privilegiada, debida en parte a la fiabilidad de sus métodos para la obtención de conocimiento, algunas personas creen que también posee autoridad práctica en los contextos donde dicho conocimiento juega un papel preponderante. Así, por consideraciones prácticas, buscamos y requerimos conocimiento fiable en áreas como la salud (Jerkert, 2013; Smith, 2012), el testimonio experto (Hansson, 2011), las políticas medioambientales (McKinnon, 2016; Torcello, 2016), la educación y formación científica (Kitcher, 1982), y el periodismo (Boykoff and Boykoff, 2004; Boykoff, 2008). Deseamos diseñar, construir y aplicar políticas sociales, currículos escolares y veredictos en las cortes a partir de nuestras creencias mejor justificadas. Tendemos a atribuirle a la ciencia un papel especial en éstas y otras tareas. De esta manera, por razones prácticas, el viejo problema de la demarcación entre la ciencia y la pseudociencia ha sido rehabilitado (Pigliucci and Boudry, 2013).

No obstante, la ciencia ha sido también cuestionada. Muestra de ello son los debates en torno a las dos culturas (Snow, 2012; Leavis, 2013) y las más recientes disputas dentro de las llamadas 'guerras de la ciencia' (Ashman and Barringer, 2001). Los críticos de la ciencia disponen también de razones tanto teóricas como prácticas para sus invectivas. A partir de un enfoque práctico e ideológico, los críticos de la ciencia en la era postcolonial afirman que es opresiva, y que "uno no puede justificar moralmente el subyugar a personas soberanas en favor de la difusión del conocimiento, (...) pues no existe algo así como un conocimiento superior, sólo diferentes conocimientos, cada uno apropiado para su situación particular" (Boghossian, 2006, 5-6). La tolerancia epistémica es la moraleja fruto de sus reflexiones. Aunque las motivaciones ideológicas tienen un papel para los críticos de la ciencia, algunos de ellos usan razones teóricas para respaldar sus afirmaciones ${ }^{2}$. Una sensación de relativismo es percibida por algunos filósofos de la ciencia y científicos al interior de los argumentos contra la presunta objetividad científica. Los argumentos que cuestionan la autoridad epistémica de la ciencia revisten distintas formas, pero a menudo lo que ponen en tela de juicio es la objetividad de la investigación científica.

1 Todas las traducciones de las citas textuales son de los autores del presente artículo.

2 Gross y Levitt (1994) realizaron un estudio sistemático de las motivaciones ideológicas que se encuentran detrás de los argumentos epistémicos de una buena parte de los críticos de la ciencia al interior de las guerras de la ciencia durante el siglo pasado. 
Si la objetividad es un ideal científico, dejarla de lado puede distorsionar y tergiversar la naturaleza y objetivos de la ciencia. Esta objetividad usualmente se interpreta como una característica de los métodos, resultados y afirmaciones científicas, las cuales - se piensa - pueden sustraerse de perspectivas particulares, compromisos de valor, sesgos comunitarios e intereses personales. No obstante, algunos críticos de la ciencia afirman que los científicos son incapaces de reunir evidencia y evaluar teorías o modelos científicos sin realizar juicios de valor contextuales; también consideran problemático su intento de alcanzar intersubjetividad plena al interior de la comunidad científica debido a sus preferencias personales y experiencias idiosincráticas. Más importante para nuestros propósitos es el hecho de que algunos críticos de la ciencia afirman que ésta es sólo un punto de vista entre muchos otros, sin algún privilegio y autoridad epistémica especial ${ }^{3}$. Epistemólogas feministas, así como otros críticos de la ciencia, "han dejado de considerar al conocimiento como una reflexión neutral y trasparente acerca de una realidad que existe independientemente de nosotros (...). Por el contrario, la mayoría acepta que todo el conocimiento es conocimiento situado, el cual refleja la posición del productor de conocimiento" (Lennon, 1997, 37).

En este artículo, nuestro objetivo es ofrecer un balance de un aspecto importante de este último debate. Argumentaremos que la concepción de la investigación científica que afirma que ésta necesariamente involucra una perspectiva o punto de vista no impone una restricción severa a su ideal de objetividad y a su autoridad epistémica. Dejamos de lado el problema de su autoridad práctica ${ }^{4}$. No obstante, sostendremos que la ciencia es objetiva y es un punto de vista privilegiado para obtener conocimiento del mundo. En otras palabras, aunque la ciencia sea un punto de vista, no es sólo un punto de vista entre otros.

Para cumplir con nuestros objetivos, primero presentamos algunas aclaraciones importantes sobre la noción de punto de vista, enfatizando su contraste con rasgos habitualmente asociados a la objetividad. En un segundo momento atendemos al concepto de objetividad científica y descartamos parcialmente un rasgo comúnmente asociado a éste, la ausencia de valores o compromisos normativos, y apostamos por una característica más global de la objetividad de la práctica científica, su fidelidad a los hechos, la cual da cuenta de mejor manera de la autoridad epistémica que le atribuimos. Por último, enfrentamos algunos desafíos particulares que se desprenden de la noción de punto de vista y amenazan rasgos de la objetividad científica vinculados con su autoridad epistémica. Argumentamos que podemos hacerles frente a muchos de estos desafíos sin aceptar consecuencias que algunos críticos de la ciencia buscan desprender del hecho de que la ciencia sea un punto de vista o involucre puntos de vista.

3 Boghossian $(2006,2)$ llama a esta tesis la 'doctrina de la validez igual': afirma que hay muchas y radicalmente distintas formas, aunque igualmente válidas, de conocer el mundo, y la ciencia sólo es una de ellas.

4 Contra algunos de los defensores y detractores de la ciencia, creemos que la relación entre la autoridad epistémica y práctica de la ciencia es menos clara de lo que suele pensarse. Feyerabend (1978) explotó este mismo punto con el objetivo — a nuestro parecer mal encaminado - de eliminar los obstáculos que intelectuales y expertos imponen a tradiciones diferentes a la suya, y de preparar la eliminación de expertos y científicos de los centros vitales de la sociedad. 


\section{Algunas aclaraciones sobre la noción de punto de vista}

El concepto de punto de vista es central en debates filosóficos en distintas áreas de la disciplina. Se discute si las representaciones que nos hacemos del mundo son relativas a un punto de vista o si podrían ser absolutas ${ }^{5}$. Algunos filósofos de la ciencia cuestionan uno de los supuestos de una cierta concepción de la investigación científica: hay un mundo y una ciencia $^{6}$. Aunque en un momento particular las teorías o modelos científicos difieran, tenderán a coincidir en un momento posterior debido a que tratan de lo mismo. Una posibilidad que no podemos descartar es que aquello acerca de lo que tratan nuestras teorías o modelos científicos no sea lo mismo ${ }^{7}$. Esto impondría restricciones adicionales a la posibilidad de una representación absoluta (unificada) de la realidad. Es ésta la arista metafísica del problema. También hay una epistémica: aunque nuestras teorías y modelos científicos traten de lo mismo, podría estarnos vedado el conocimiento de representaciones absolutas ${ }^{8}$. Una última arista es semántica: ¿qué determina el contenido de nuestras representaciones? A diferencia de una representación absoluta, en una perspectival el punto de vista realiza una contribución indispensable al contenido de la representación (Moore, 1987, 2).

Antes de ocuparnos de aspectos específicos de estos problemas, presentamos aclaraciones sobre el sentido en que puede decirse que la ciencia es un punto de vista. En los siguientes apartados nos ocuparemos de precisar en qué medida esto impone restricciones sobre su ideal de objetividad.

La expresión 'punto de vista' se usa ordinariamente de una manera vaga. Suele emplearse como un prefijo al inicio de una oración: 'desde $x$ punto de vista', quizá para indicar al oyente o lector que lo que sigue está de algún modo restringido o limitado, y como antónimo a 'de manera categórica' o 'exhaustivamente' (Hautamäki, 1986, 63-65). Así, que a una oración le anteceda 'desde $x$ punto de vista' insinúa al interlocutor que no se han tomado en cuenta todos los aspectos de una cuestión o que no se han considerado todas las posibilidades relevantes dependiendo de diversos factores: los intereses, los objetivos, los valores y las presuposiciones del hablante (Lehtonen, 2011, 237). A pesar de estas consideraciones conceptuales iniciales, podemos dar cuenta de manera más sistemática de algunos aspectos epistémicos del concepto.

Para Lehtonen (2011, 246-250), el concepto de punto de vista se refiere a una consideración racional que tiene muchos componentes, algunos de los cuales refieren al sujeto que observa, otros a las herramientas e instrumentos de observación y otros al objeto que es observado. Las variables relacionadas con el sujeto de observación brindan los siguientes componentes: (a) el sujeto o el tipo de sujeto que observa, (b) sus intereses, objetivos y valores, (c) la postura psicológica o actitud del sujeto, (d) el conocimiento relevante de

5 Este problema fue tratado con cierto detalle por Bernard Williams (1978, 64-5; 1985, 138), quien ofreció razones a favor de la posibilidad de representaciones absolutas. Una defensa más reciente, inspirada en Williams, es de la A.W. Moore (1987), quien posteriormente (1997) desarrolló de manera mucho más sistemática su defensa.

6 André Kukla (2008) rechaza el argumento de Rescher (1985; 1998) de 'un mundo, una ciencia' debido a que parte de supuestos contenciosos.

7 Esta posibilidad ha sido explorada por los pluralistas ontológicos (e.g., Dupré, 1993; Cartwright, 1999).

8 Una de las defensas más conocidas de la posibilidad de tener conocimiento objetivo de representaciones absolutas, aunque admite aspectos perspectivales de la realidad, fue realizada por Thomas Nagel (1986). 
fondo y las expectativas del sujeto, y (e) el contexto social, cultural e histórico del sujeto. Las variables relacionadas con las herramientas de observación brindan los siguientes componentes: (f) el aparato conceptual usado por el sujeto, (g) el método de observación y (h) la base de observación o los datos. Por último, las variables relacionadas con el objeto observado brindan los siguientes componentes al concepto: (i) el objeto o el enfoque del objeto de un punto de vista y (j) las características o propiedades observables del objeto. Podemos incorporar estas variables, condensadas en un solo índice, mediante el siguiente operador oracional:

\section{$P V(s) p$ : desde el punto de vista de $s, p$.}

Donde ' $P V$ ' es un operador que asigna valuaciones a fórmulas, a partir de argumentos para ' $s$ ', el cual contiene los índices arriba enlistados en los incisos (a)-(j); como es habitual, ' $p$ ' designa a una oración (simple o compleja) susceptible de ser verdadera o falsa. Como señala Lehtonen $(2011,250)$, el operador del punto de vista no es veritativo-funcional, debido a que el valor de verdad de ' $P V(s) p$ ' no está determinado por el valor de verdad de ' $p$ ', como resultado de que los puntos de vista se representan como contextos intensionales que incluyen creencias, conocimiento e intereses. Adicionalmente, dado este análisis de los elementos del concepto, puede apreciarse que la mayoría de ellos se relacionan o dependen del sujeto del punto de vista, así como de sus intereses.

A partir de este análisis de los elementos involucrados en el concepto de punto de vista, ¿en qué sentido es la ciencia un punto de vista? Mencionaremos, sólo para dejarlos de lado, algunos aspectos de la noción de punto de vista hasta ahora examinada que no parecen representar desafíos significativos para la objetividad científica. Nadie negaría que la ciencia involucra un punto de vista en los siguientes sentidos: (1) cualquier investigación científica particular es incompleta con respecto al punto de vista adoptado, debido al hecho de que su rango está determinado por los temas elegidos en la investigación y los intereses de los investigadores; (2) cualquier investigación científica está limitada por factores espacio-temporales e histórico-sociales; (3) cualquier investigación científica es realizada por seres humanos concretos que difieren en su competencia, instrucción y tipo; (4) las investigaciones científicas son a menudo producidas colaborativamente por miembros de un grupo social; (5) en ocasiones los valores político-sociales influyen en la manera en la que los investigadores realizan su trabajo; (6) en ocasiones las investigaciones científicas están sesgadas por el trasfondo de valores de los investigadores, lo que no pocas veces les lleva a creer afirmaciones para las que no disponen de evidencia. Estos supuestos son aceptados de manera general y forman parte de la imagen clásica del conocimiento, la cual se remonta hasta Aristóteles (Boghossian, 2006, 19-20). Estos aspectos, en los que la ciencia constituye un punto de vista, no amenazan el ideal de objetividad de la práctica científica debido a que ésta suele disponer de mecanismos para neutralizar los sesgos y la parcialidad que introducen las personas, su situación, sus intereses y valores en la investigación.

Otros componentes del concepto de punto de vista pueden amenazar seriamente el ideal de objetividad científica, pero antes debemos señalar algunas características de la objetividad que suele atribuirse a la ciencia. 


\section{Objetividad científica y compromisos normativos}

Pensemos por un momento que nuestra sociedad está en ciernes. Nuestras instituciones son inmaduras y buscamos establecer sólidas bases para el desarrollo económico y social de nuestra pequeña población. Si somos mujeres y hombres reflexivos, perteneceremos a un pequeño subconjunto de personas que pueden ser definidas por su búsqueda del conocimiento. Puede que se nos ocurra fundar una pequeña asociación de investigación en la que colaborativamente busquemos el conocimiento de aquello que nos es tanto interesante como urgente. ¿A quiénes hemos de aceptar en nuestra cofradía? Puede ocurrírsenos descartar a los comerciantes y artesanos, pues sus preocupaciones mundanas pueden corromper sus juicios acerca de lo que constituye verdadero conocimiento. No obstante, con el tiempo nos percataremos de que incluso las personas mejor educadas pueden falsear la información, reportar experimentos que nunca realizaron, y apoyar conjeturas sin fundamento debido a sus pasiones políticas y personales. No obstante, también podemos seguir honrando el ideal de objetividad de nuestra asociación a pesar de los obstáculos 9 . En un sentido muy básico, y quizá poco interesante, el ideal de objetividad de la ciencia puede ser descrito a partir de nuestro escenario imaginado: la ciencia busca evitar la parcialidad que amenaza a cualquier búsqueda de conocimiento. Como mencionamos anteriormente, la ciencia dispone de mecanismos internos para neutralizar estos tímidos desafíos. Esta burda caracterización puede sernos útil para señalar un posible rasgo inicial que permita capturar la noción de objetividad científica: la ausencia de valores o compromisos normativos.

Suele señalarse que la objetividad en ciencia, que da lugar a su especial autoridad epistémica, radica en que no está contaminada de valores morales, políticos y sociales. No obstante, nadie negaría que los valores de los investigadores de hecho afectan la elección del problema o los problemas científicos que se investigarán y las posibles aplicaciones de los resultados de la investigación. Estas afectaciones, sin embargo, no resultan problemáticas para una noción interesante de objetividad científica. Aunque el conjunto de problemas que los científicos se propongan investigar y las aplicaciones que deseen desarrollar sean determinadas por valores, esto no constituye un obstáculo para que sean capaces de discriminar entre respuestas correctas e incorrectas acerca de esos problemas, y puedan juzgar qué aplicaciones son más eficientes que otras para lograr sus objetivos iniciales. No sucede así con otras dos posibles afectaciones. Valores de diversa índole pueden contaminar la manera en la que los científicos reúnen evidencia en relación a su problema de investigación, y su posible aceptación de hipótesis y teorías científicas.

Así, sin alguna clase de compromiso con algunos valores, la investigación científica sería imposible. Esto se ve con mayor claridad en el caso de las ciencias sociales. La investigación social está orientada a la implementación de políticas sociales (i.e., a formas de intervenir para modificar o mejorar la sociedad). Si las ciencias sociales no cumplieran esta función, no responderían a nuestro interés por comprender los fenómenos sociales. Pero tales políticas,

9 Este escenario imaginado es una reconstrucción de la historia de la fundación de la Royal Society. Kitcher $(2001,29)$ lo usa de este modo para motivar un sentido muy básico de objetividad que está presente en la investigación científica, y que se relaciona directamente con los elementos que consideramos no amenazadores del ideal de objetividad que están presentes en el concepto de punto de vista. 
y los valores que involucran, pueden afectar la objetividad de la investigación, poniendo en duda su carácter científico y volviéndola inútil como base común para personas con intereses distintos. En este punto, parece que enfrentamos un dilema (Risjord, 2014, 14):

1. Las ciencias sociales o bien (i) involucran valores o bien (ii) están libres de valores.

2. Si (i) involucran valores, entonces no son objetivas y son imposibles como (a) ciencias.

3. Si (ii) están libres de valores, no contribuyen a comprender la sociedad y son imposibles como (b) sociales.

Por tanto, las (a) ciencias (b) sociales no son posibles.

No obstante, la segunda afirmación del argumento anterior depende de un supuesto cuestionable que considera que la objetividad implica ausencia de valores. En otras palabras, da por supuesto que la ciencia sólo puede ser objetiva si los valores no juegan ningún papel en ella. En contra de este supuesto, como sugerimos anteriormente, parece claro que no toda afectación de valores en la investigación compromete la objetividad científica. Adicionalmente, en el caso particular de las ciencias sociales, si hemos de reconocer su posibilidad, debemos reconsiderar este supuesto y admitir que (al menos, algunos de) los valores pueden jugar (al menos, algunos) papeles en la investigación científica.

Dos cuestiones surgen si aceptamos que al menos algunos valores pueden incidir en la investigación sin comprometer su objetividad: (i) ¿qué tipo de valores juegan un papel en la ciencia?, y (ii) ¿cómo afectan estos valores la práctica científica? Así, para reconocer varias respuestas a estas preguntas conviene distinguir entre distintos tipos de valores y diversas maneras en que afectan a la práctica científica. A la luz de estas distinciones, examinaremos qué consecuencias tienen para la objetividad de la ciencia.

En un sentido importante, toda investigación científica involucra valores. Pero no toda influencia valorativa en la práctica científica afecta su objetividad: algunos valores parecen contribuir a la objetividad, mientras que otros, en efecto, pueden socavarla. Así, resulta necesario distinguir entre dos tipos de valores: epistémicos y no epistémicos. Los valores epistémicos son aquellos que contribuyen a la objetividad, por lo que fomentan la buena práctica científica: alientan a los investigadores a buscar teorías bien confirmadas, simples y explicativas. Al elegir entre métodos para investigar, los científicos se sirven de valores para elegir entre diferentes maneras de lograr buenos resultados científicos. Por su parte, los valores no epistémicos tienen otros objetivos, quizá morales o políticos, por lo que pueden generar resultados tendenciosos o sesgados, lo que afectaría la objetividad de la investigación al promover, entre otras cosas, ciertos ideales políticos o morales.

Por otra parte, en la práctica científica, siempre intervienen ambos tipos de valores: los científicos son seres humanos y sus actividades se producen en una sociedad que puede promoverlas u obstaculizarlas. No obstante, podemos distinguir dos papeles de los valores que afectan a la investigación (Longino, 1990). Algunos valores son constitutivos, en el sentido de que definen cómo se lleva a cabo la práctica científica. Otros valores son contextuales, en el sentido de que afectan que la práctica se lleve a cabo o no, sin definir cómo se realiza.

Con estas distinciones en mente, podemos mitigar la tesis de que la objetividad requiere ausencia de valores o compromisos normativos. Una tesis moderada sería la siguiente: la 
ciencia es objetiva cuando sólo los valores epistémicos son constitutivos de la práctica y los valores no epistémicos siempre son sólo contextuales (Risjord, 2014, 19) ${ }^{10}$.

Si la objetividad de la práctica científica no puede ser sencillamente caracterizada a partir de su ausencia de valores o compromisos normativos, ¿cómo dar cuenta de ella? La de objetividad no es una noción filosófica unívoca y, como muchos otros conceptos filosóficos, suele estar cargada de distintas dimensiones, cada una de ellas focalizada en contra de algunos peligros. Por ello, Crasnow (2006) distingue tres sentidos de objetividad:

(a) Ausencia de sesgos: el método no es tendencioso, no favorece hipótesis por razones distintas a la evidencia.

(b) Intersubjetividad: la hipótesis puede ser examinada por más de una persona.

(c) Confiabilidad: la hipótesis es producto de métodos que dan resultados probablemente verdaderos.

Es cierto que estos diversos sentidos no siempre coinciden. Mientras la parcialidad y la no neutralidad, en apariencia opuestas a una noción general de objetividad científica, amenazan el sentido (a) de objetividad, son compatibles con la investigación objetiva en los sentidos (b) y (c). Así, incluso si tratan acerca de valores o buscan promoverlos, las investigaciones podrían ser objetivas.

Lidiar con otras cuestiones en torno a la objetividad de la ciencia requiere comprender cómo los valores podrían jugar un papel en la construcción de teorías, en la formación de conceptos y en la manera en que las hipótesis se ponen a prueba.

Otro rasgo, quizá el más fundamental, de la noción filosófica de objetividad involucra fidelidad a los hechos. Se asume que la ciencia es capaz de representar los hechos tal como son, con independencia de nuestras preferencias y valoraciones sobre dichos hechos. Esta característica, en caso de poseerla, daría cuenta de la fiabilidad de los métodos científicos para la obtención de conocimiento, lo que, como señalábamos al inicio, parece estar en el trasfondo de su autoridad epistémica.

En la siguiente sección examinaremos maneras específicas en las que la noción de punto de vista en la práctica científica plantea problemas para esta forma de objetividad.

\section{Los puntos de vista como desafío epistémico a la objetividad científica}

Para apreciar con claridad el desafío teórico de los puntos de vista hacia la objetividad científica, entendida como fidelidad a los hechos, podemos considerar un patrón común de argumentos que la ponen en entredicho. Una manera interesante de capturar este desa-

10 Las ciencias sociales merecen una consideración especial con respecto a esta versión moderada de la tesis de la ausencia de valores o compromisos normativos, pues no parecen cumplir con este requisito incluso en su versión más modesta. Por una parte, los valores no epistémicos parecen influir en la manera en la que se justifican las hipótesis en ciencias sociales (no son imparciales); por otra parte, pueden ser parte del contenido de las teorías de las ciencias sociales (no son neutrales). En ambos casos, desempeñarían un papel constitutivo en la práctica científica. ¿Significa esto que las ciencias sociales no pueden ser objetivas? Para responder a esta interrogante es preciso atender a las razones por las que se afirma que las ciencias sociales no pueden ser imparciales o neutrales (Risjord, 2014, 20). 
fío consiste en tomar como molde la estructura de un conocido problema en filosofía de la ciencia: la tesis de la 'subdeterminación de las teorías a partir de la evidencia'"1. De manera esquemática, el problema se plantea al reconocer que incluso las teorías científicas empíricamente más exitosas tienen competidoras que, aunque son incompatibles, resultan igualmente aceptables a partir de la evidencia. Esto significa que, incluso si tomamos el éxito empírico de las teorías como criterio para decidir racionalmente sobre su aceptación, este criterio es insuficiente para determinar que una teoría, en lugar de sus competidoras, es (aproximadamente) verdadera o fiel a los hechos (Lyons, 2016, 572-573). Antes de detenernos a examinar cómo la noción de punto de vista que hemos caracterizado hasta ahora puede ser vertida sobre este molde, conviene que resaltemos algunos aspectos cruciales del desafío general.

Un supuesto apenas cuestionado por quienes participan en estos debates es que la elección de teorías debería estar, al menos en parte, guiada por una estimación de su éxito empírico. En términos generales, una teoría es empíricamente exitosa cuando dispone de una amplia capacidad para ajustarse adecuadamente a los datos disponibles y hacer predicciones novedosas, en ocasiones sorprendentes, sobre fenómenos observables. Decimos que una teoría es confirmada por la evidencia cuando los datos empíricos respaldan la estimación de que la teoría se encuentra en posesión de estas capacidades; puede haber variaciones significativas tanto en la amplitud como en la precisión con la que se estima que manifiesta poseerlas. En consecuencia, además del juicio puramente cualitativo de que una teoría está confirmada sin más, efectuamos juicios sobre el grado o la extensión en los que la teoría es avalada por la evidencia.

Le tesis de la subdeterminación encarna la afirmación de que varias teorías están igualmente confirmadas por conjuntos específicos de datos empíricos. Esto puede significar simplemente que logran, en algún grado, adecuarse a los datos o generar predicciones correctas. Que esto de hecho ocurre difícilmente puede cuestionarse, pero no es especialmente problemático para la objetividad entendida como fidelidad a los hechos. En tanto haya diferencias en el grado o extensión en los que varias teorías son confirmadas, la estimación de su éxito empírico sigue ofreciendo una valiosa orientación sobre qué teorías deberían preferirse por encima de otras. Sin embargo, la afirmación de que varias teorías están igualmente confirmadas o son empíricamente equivalentes puede también entenderse como indicando que dos o más teorías son respaldadas con la misma extensión o grado (o una combinación ponderada de ambos factores) por conjuntos específicos de evidencia. Esta afirmación es más contenciosa y requiere ser examinada con mayor detenimiento. No obstante, en caso de que fuese correcta, de manera directa parece entrar en conflicto con la objetividad como fidelidad a los hechos. Si varias teorías son igualmente exitosas en sus logros empíricos, no parece haber razones para sostener que la estimación de su éxito empírico nos orienta sobre qué teoría preferir.

11 Al final de su libro Science without Laws, Ronald Giere (1999, 237-241) sugiere que la subdeterminación de las teorías por la evidencia suele ser tomada como una premisa, en argumentos explícitos o implícitos, para socavar de diversas formas la objetividad científica. No obstante, Giere no elabora, como lo hacemos a continuación, la forma en que el aspecto perspectival se introduce en la tesis de la subdeterminación para amenazar la autoridad epistémica de la práctica científica. 
Dicho lo anterior, es importante enfatizar que una condición adicional para que la equivalencia empírica genere problemas a la objetividad científica es que las teorías igualmente confirmadas se encuentren en competencia. Muchas alternativas a una teoría específica no rivalizan con ella: podrían ser complementos o incluso meras variaciones inocuas de la teoría original. Para que el reto de la subdeterminación a la objetividad científica constituya un problema, las alternativas empíricamente equivalentes deben ser teorías distintas, mutuamente incompatibles, en el sentido de que la aceptación de una de ellas excluya la posibilidad de aceptar otras.

En resumen, la noción de punto de vista puede constituir un desafío significativo a la objetividad científica entendida como fidelidad a los hechos siempre que se cumplan las siguientes condiciones: (A) debido a un rasgo de los puntos de vista, puede decirse que varias teorías son empíricamente equivalentes; (B) las teorías en cuestión son incompatibles; y (C) el criterio para elegir cuál teoría aceptar depende crucialmente de su éxito empírico. Aunque las últimas dos condiciones pueden generar sospechas, en lo que sigue asumiremos que se cumplen. Nos concentraremos en discutir las formas más plausibles en que parecería satisfacerse la primera de estas condiciones. Examinaremos tres maneras distintas e independientes en las que puede decirse que dos (o más teorías) son empíricamente equivalentes debido a la intervención de un punto de vista. Para ello recuperaremos las intuiciones, que presentamos en la primera sección, de que los puntos de vista involucran: variaciones en el conocimiento de fondo de los sujetos, las posturas psicológicas o actitudes de los sujetos y los aparatos conceptuales que emplean los sujetos.

(I) El conocimiento de fondo de los sujetos podría producir que distintas teorías se estimen como igualmente confirmadas por la misma evidencia al sólo considerar cuán empíricamente exitosas son estas teorías. Esto es reconocido incluso cuando se utilizan estándares bastante explícitos y precisos para especificar en qué consiste que una pieza de evidencia confirme una teoría ${ }^{12}$. Sin embrago, el quid de este asunto puede apreciarse también de manera informal. El grado en que la evidencia incrementa la credibilidad de una hipótesis (es decir, la confirma) depende de manera importante de qué tan creíble se consideraba a la hipótesis en primer lugar. Y esto puede diferir entre sujetos en los que se presentan variaciones en el conocimiento de fondo: mientras uno asume que la hipótesis $H$ es prima facie muy razonable, otros pueden tener convicciones distintas debido a que no comparten un núcleo sustantivo de creencias con aquél. De este modo, incluso si dos científicos toman en cuenta la misma evidencia y emplean los mismos procedimientos racionales para evaluarla, sus juicios acerca de cuál de ellas es preferible pueden diferir. Para científicos con distintos puntos de vista, entendidos como conocimiento de fondo, dos alternativas teóricas en competencia pueden estar igualmente confirmadas. Si ninguno de los grados de creencia iniciales de los sujetos ha de considerarse privilegiado (¿y por qué habría de serlo?), entonces parece claro que tampoco debería serlo ninguno de los juicios sobre confirmación a partir de la evidencia.

$12 \mathrm{Al}$ discutir estos problemas pensamos primariamente en teorías robustas de la confirmación, tales como las varias alternativas bayesianas. No emplearemos detalles excesivamente técnicos de estas teorías; aunque son versátiles para plantear estas discusiones, no asumimos que ésta sea la única manera de presentar los puntos relevantes. Para un panorama ilustrativo del bayesianismo pueden consultarse Skyrms (2000); Howson y Urbach (2005); Douven (2011); pero también Williamson (2017). 
¿Puede desmantelarse esta amenaza a la objetividad científica? En ocasiones se ha sugerido que deben existir restricciones sobre la credibilidad inicial de las hipótesis ${ }^{13}$. Sin embargo, a la fecha no existe consenso sobre cuáles ni qué tan severas podrían ser estas restricciones. En todo caso, los prospectos de ofrecer una solución definitiva a esta versión del problema al limitar los puntos de vista que pueden considerarse aceptables no son muy alentadores. Aunque este diagnóstico puede parecer desconcertante, hay buenas noticias en el horizonte para una concepción robusta de objetividad científica, si tomamos en cuenta el efecto dinámico de reunir más evidencia. Incluso si hay bastante libertad en la credibilidad inicial que varios sujetos pueden asignar a una hipótesis y ésta afecta los juicios sobre en qué medida es confirmada por la evidencia - en tanto el procedimiento de asimilar piezas de información empírica se mantenga constante-, el efecto de los grados de creencia iniciales se desvanece a la larga a medida que se incorpora nueva evidencia. De este modo, investigadores que toman como punto de partida distinto conocimiento de fondo tienden a converger, a largo plazo, en el grado exacto en que una misma hipótesis es confirmada por la evidencia.

(II) La postura o actitud psicológica de los sujetos también puede ocasionar que distintas teorías se ponderen como igualmente confirmadas por la misma evidencia al tomar en cuenta sólo sus credenciales empíricas. Aun cuando le asignaran la misma credibilidad inicial a diversas hipótesis, distintos sujetos podrían emitir diferentes dictámenes sobre qué tan confirmada está una teoría. Esto ocurriría especialmente si consideramos a la postura psicológica o actitud del sujeto como su disposición a cambiar de formas específicas sus grados de creencia a partir de la evidencia. Cuando hay diferencias en la manera en que razonan los científicos a partir de los mismos fragmentos de evidencia, no es sorprendente que se desvíen sus veredictos sobre qué teoría está siendo apoyada y en qué medida; esto ofrece una nueva razón para esperar que distintas alternativas sean igualmente respaldadas por la misma evidencia.

Aunque los peligros de esta línea argumentativa han cautivado a varios pensadores, es preciso señalar que la sugerencia esbozada en el párrafo anterior es propensa a generar confusiones. Esto se debe a que la noción de punto de vista como postura psicológica o actitud del sujeto captura dos aspectos interrelacionados, pero diferentes, de la manera que los seres humanos razonan a partir de la evidencia: uno descriptivo y otro normativo. La cuestión descriptiva se conecta con la manera en que de hecho razonan las personas; ésta no siempre es adecuada o racional y depende de factores que a menudo somos incapaces de reconocer o controlar conscientemente. Determinar qué tanta variación puede presentarse entre individuos sobre estos rasgos psicológicos es un asunto empírico, sobre el que no habría de asumirse que una respuesta meramente especulativa ofrezca bases concluyentes. Por otra parte, la cuestión normativa sobre cómo deberíamos razonar a partir de la evidencia podría tener una respuesta independiente de las variaciones contingentes entre individuos específicos: quizá, en situaciones concretas, alguno de ellos simplemente razona de manera errónea. En todo caso, la prometedora (aunque hasta ahora incompleta) réplica de las ciencias cognitivas y las teorías de la confirmación es que, en ambos sentidos, los puntos

13 Algunas sugerencias célebres desde la perspectiva bayesiana son que la credibilidad inicial de hipótesis debería estar restringida por ciertos principios; por ejemplo, Lewis (1980), Maher (1993), Williamson (2017). 
de vista entendidos como posturas psicológicas o actitudes de los sujetos admiten mucha menos variación (racional) de la que parecería existir a primera vista. Muchas diferencias idiosincrásicas entre individuos parecen depender, más bien, de los puntos de vista entendidos como conocimiento de fondo.

(III) Un último desafío se desprende de examinar cómo los aparatos conceptuales pueden propiciar que diferentes teorías estén igualmente confirmadas por la misma evidencia, si uno se restringe a evaluar sus méritos empíricos. Incluso sujetos que asimilan de la misma manera la nueva información y asignan la misma credibilidad inicial a diversas hipótesis pueden diferir en sus juicios sobre la confirmación de una teoría, en caso de que empleen diferentes marcos conceptuales. Puesto que éstos fijan las maneras en las que la nueva información empírica es representada, así como las relaciones lógicas y epistémicas entre hipótesis previamente aceptadas, esta forma de concebir a los puntos de vista parece inaugurar una nueva ruta hacia la subdeterminación.

La principal inquietud que da origen a esta versión del problema parece tener sus raíces en las observaciones kuhnianas sobre la incomensurabilidad entre paradigmas (Kuhn, 1962, chap. 10). Mucha tinta se ha derramado en torno a cuál es la manera correcta de entender estas observaciones y cuáles serían sus repercusiones. No intentaremos reproducir esa discusión aquí. Lo que sí puede señalarse a favor de la objetividad científica como fidelidad a los hechos es que no parece que esta nueva amenaza pueda separarse nítidamente de los dos desafíos que examinamos con anterioridad. $\mathrm{O}$, por lo menos, tal deslinde no puede realizarse garantizando que las teorías en competencia son genuinamente distintas. De entrada, parece poco plausible que científicos que investigan algún conjunto de fenómenos empleando aparatos conceptuales distintos puedan siquiera compartir los mismos grados de credibilidad inicial para diversas hipótesis; ¿cómo podrían, si sus aparatos conceptuales son radicalmente distintos? Por otra parte, si las conexiones lógicas y epistémicas que se establecen entre hipótesis varían, no parece claro que razonen de manera análoga; de modo que podría ser su razonamiento, y no sus aparatos conceptuales, lo que explica las diferencias entre sus juicios de confirmación. Pero ya hemos ofrecido respuestas para estos argumentos de subdeterminación. Una veta aún abierta consiste en suponer que sus aparatos conceptuales, aunque distintos, permiten una comparación entre hipótesis y sus relaciones lógico-epistémicas. Manteniendo constantes estos factores, podría asegurarse que sólo el aparato conceptual es responsable de las diferencias en la evaluación de hipótesis; sin embargo, no parece claro que podamos decir sin más que tales evaluaciones son diferentes: las teorías sobre las que se aplican podrían ser las mismas, arropadas bajos distintos atavíos representacionales. Finalmente, uno podría pensar que este último reto es el producto de integrar el efecto acumulativo todos estos aspectos de los puntos de vista. Si eso ha de considerarse una amenaza a la objetividad científica, no es claro cómo podría especificarse en un argumento sistemático; en efecto, ésa parece haber sido la descorazonadora lección de la extensa discusión sobre la inconmensurabilidad entre teorías.

El balance de la discusión precedente es que, si consideramos sólo los argumentos a partir de subdeterminación que esbozamos al inicio de esta sección, la noción de punto de vista es compatible con una buena dosis de objetividad, entendida como fidelidad a los hechos. Incluso si hay diferencias entre puntos de vista, en cualquiera de los tres sentidos aquí examinados, puede haber criterios claros para preferir aquellas teorías para las que tenemos buenas bases racionales y es posible que sean, en aspectos importantes, aproximadamente verdaderas. 


\section{Conclusiones}

En este artículo hemos examinado en qué sentido la ciencia es o involucra puntos de vista. Algunos críticos de la ciencia suelen apoyarse en esta posibilidad para negar privilegios epistémicos a la práctica científica. Si la ciencia tiene alguna autoridad epistémica en absoluto, debido a sus presuntos métodos fiables en la obtención de conocimiento, una cuestión inesquivable será averiguar en qué medida el hecho de que la ciencia sea un punto de vista, o involucre puntos de vista, restringe sus pretensiones de objetividad. Por esta razón, en la segunda sección del artículo realizamos algunas clarificaciones de índole conceptual e investigamos algunos componentes de la noción de punto de vista. Sugerimos que algunos de ellos son inofensivos, en tanto nadie negaría que la práctica científica está limitada por diversos factores propios de sus practicantes y su contexto, sin que ello afecte directamente la posibilidad de alcanzar objetividad en la investigación. Otros factores, no obstante, pueden afectar a las pretensiones de objetividad de la práctica científica si son usados en cierto tipo de argumentos. En la tercera sección del artículo analizamos algunas características o rasgos de la noción de objetividad científica. Descartamos parcialmente la característica de ausencia de valores o compromisos normativos, debido a que su presencia es inherente a la práctica científica y no todo compromiso valorativo afecta la objetividad, pues valores específicamente epistémicos la promueven. En la cuarta sección, examinamos desafíos más específicos que se desprenden de la noción de punto de vista y amenazan rasgos de la objetividad científica vinculados con su autoridad epistémica. Concluimos que muchos de estos desafíos pueden enfrentarse sin hacer concesiones importantes a los críticos de la objetividad científica.

\section{Referencias}

Ashman, K. / Barringer, P. (eds.) (2001), After the Science Wars: Science and the Study of Science, Nueva York, Routledge.

Boghossian, P. (2006), Fear of Knowledge. Against Relativism and Constructivism, Nueva York, Oxford University Press.

Boykoff, M. (2008), "Lost in Translation? United States Television News Coverage of Anthropogenic Climate Change, 1995-2004", Climatic Change, 86, pp. 1-11.

Boykoff, M. / Boykoff, J. (2004), "Balance as Bias: Global Warming and the U.S. Prestige Press", Global Environmental Change, 14, pp. 125-136.

Cartwright, N. (1999), The Dappled World: A Study of the Boundaries of Science, Cambridge, Cambridge University Press.

Crasnow, S. (2006), "Feminist Contributions to Anthropology and Sociology", en: Turner, S. / Risjord, M. (eds.), Philosophy of Anthropology and Sociology, Amsterdam, Elsevier.

Douven, I. (2011), "Relativism and Confirmation Theory", en: Hales, S.D. (ed.), A Companion to Relativism, Nueva York, Blackwell, pp. 242-263.

Dupré, J. (1993), The Disorder of Things: Metaphysical Foundations of the Disunity of Science, Cambridge, Harvard University Press.

Feyerabend, P. (1978), Science in a Free Society, Londres, New Left Books.

Giere, R. (1999), Science without Laws, Chicago, The University of Chicago Press. 
Gross, P. / Levitt, N. (1994), Higher Superstition. The Academic Left and its Quarrels with Science, Baltimore, The Johns Hopkins University Press.

Hansson, S.O. (2011), "Philosophy in the Defence of Science", Theoria, 77(1), pp. 101-103. Hautamäki, A. (1986), "Points of View and Their Logical Analysis", Acta Philosophica Fennica, 41, pp. 1-156.

Howson, C. / Urbach, P. (2005), Scientific Reasoning: The Bayesian Approach, $3^{\text {rd }}$ ed., Chicago, Open Court.

Jerkert, J. (2013), "Why Alternative Medicine Can be Scientifically Evaluated. Countering the Evasions of Pseudoscience", en: Pigliucci, M. / Boudry, M. (eds.), Philosophy of Pseudoscience. Reconsidering the Demarcation Problem, Chicago, The University of Chicago Press, pp. 305-320.

Kitcher, P. (1982), Abusing Science. The Case Against Creationism, Cambridge, MIT Press. Kitcher, P. (2001), Science, Truth and Democracy, Nueva York, Oxford University Press.

Kuhn, T. S. (1962), The Structure of Scientific Revolutions, Chicago, The University of Chicago Press.

Kukla, A. (2008), “The One World, One Science Argument”, British Journal for the Philosophy of Science, 59(1), pp. 73-88.

Leavis, F.R. (2013), Two Cultures? The Significance of C.P. Snow, Nueva York, Cambridge University Press.

Lehtonen, T. (2011). "The Concept of a Point of View”, SATS, 12(2), pp. 237-252.

Lennon, K. (1997), "Feminist Epistemology as Local Epistemology", Proceedings of the Aristotelian Society, Supplementary Volume 71, pp. 37-54.

Lewis, D.K. (1980), “A Subjectivist's Guide to Objective Chance”, en: Jeffrey, R. C. (ed.), Studies in Inductive Logic and Probability, vol. 2, Berkeley, University of California Press, pp. 263-293.

Longino, H. (1990). Science as Social Knowledge: Values and Objectivity in Scientific Inquiry, Princeton, Princeton University Press.

Lyons, D. (2016), “Scientific Realism”, en: Humphreys, P. (ed.), The Oxford Handbook of Philosophy of Science, Nueva York, Oxford University Press, pp. 564-584.

Maher, P. (1993), Betting on Theories, Cambridge, Cambridge University Press.

McKinnon, C. (2016), "Should We Tolerate Climate Change Denial?", Midwest Studies in Philosophy, 40(1), pp. 205-216.

Moore, A.W. (1987), "Points of View", The Philosophical Quarterly, 30(146), pp. 1-20.

Moore, A.W. (1997), Points of View, Nueva York, Oxford University Press.

Nagel, T. (1986), The View from Nowhere, Nueva York, Oxford University Press.

Pigliucci, M. / Boudry, M. (eds.) (2013), Philosophy of Pseudoscience. Reconsidering the Demarcation Problem, Chicago, Chicago University Press.

Rescher, N. (1985), "Extraterrestrial Science”, en: Regis E. (ed.), Extraterrestrials: Science and Alien Intelligence, Cambridge, Cambridge University Press, pp. 83-116.

Rescher, N. (1998), Complexity: A Philosophical Overview, Nueva Brunswick, Transaction Publishers.

Risjord, M. (2014), Philosophy of Social Science. A Contemporary Introduction, Nueva York, Routledge. 
Skyrms, B. (2000), Choice and Chance. An Introduction to Inductive Logic, $4^{\text {th }}$ ed. Ontario, Wadsworth.

Smith, K. (2012), “Against Homeopathy. A Utilitarian Perspective”, Bioethics, 26(8), pp. 398-409.

Snow, C.P. (2012), The Two Cultures: And a Second Look: An Expanded Version of The Two Cultures and the Scientific Revolution, Nueva York, Cambridge University Press.

Torcello, L. (2016), "The Ethics of Belief, Cognition, and Climate Change Pseudoskepticism: Implications for Public Discourse", Topics in Cognitive Science, 8(1), pp. 19-48.

Williams, B. (1978), Descartes: The Project of Pure Enquiry, Hertfordshire, Harvester Press.

Williams, B. (1985), Ethics and the Limits of Philosophy, Cambridge, Harvard University Press.

Williamson, J. (2017), Lectures on Inductive Logic, Nueva York, Oxford University Press. 
